PROCEEDINGS OF THE AMERICAN MATHEMATICAL SOCIETY

Volume 124, Number 10, October 1996

\title{
A CLASS OF COMPLETE SECOND ORDER LINEAR DIFFERENTIAL EQUATIONS
}

\author{
HIROKAZU OKA
}

(Communicated by Palle E. T. Jorgensen)

\begin{abstract}
This paper is concerned with a class of complete second order linear differential equations in a Banach space. We show the existence and uniqueness of classical solutions of
\end{abstract}

$$
\left\{\begin{array}{l}
u^{\prime \prime}(t)=A(t) u^{\prime}(t)+B(t) u(t)+f(t) \text { for } t \in[0, T] \\
u(0)=x \text { and } u^{\prime}(0)=y
\end{array}\right.
$$

\section{INTRODUCTION}

In this paper we study complete second order linear differential equations

$$
\left\{\begin{array}{l}
u^{\prime \prime}(t)=A(t) u^{\prime}(t)+B(t) u(t)+f(t) \text { for } t \in[0, T] \\
u(0)=x \text { and } u^{\prime}(0)=y
\end{array}\right.
$$

in a Banach space $X$. Here $\{A(t): t \in[0, T]\}$ is a family of closed linear operators in $X$ with the common domain $D$ of $A(t)$ and $\{B(t): t \in[0, T]\}$ is a family of linear operators in $X$ with $D \subset D(B(t))$ for $t \in[0, T]$.

In this case, as pointed out in [5], (SE) is closely related to the first order abstract Cauchy problem

$$
\left(\mathrm{CP} ; u_{0}, f\right) \quad\left\{\begin{array}{l}
u^{\prime}(t)=A(t) u(t)+f(t) \text { for } t \in[0, T] \\
u(0)=u_{0} .
\end{array}\right.
$$

Recently, Tanaka [10] has studied the problem $\left(\mathrm{CP} ; u_{0}, f\right)$ above in the case where a family $\{A(t): t \in[0, T]\}$ of closed linear operators in $X$ satisfies all conditions which are usually referred to as the "hyperbolic" case except for the density of the common domain $D$ of $A(t)$ and shown the existence and uniqueness result of classical solutions of $\left(\mathrm{CP} ; u_{0}, f\right)$.

The purpose of this paper is to prove the existence and uniqueness of classical solutions of (SE) on the basis of the results on $\left(\mathrm{CP} ; u_{0}, f\right)$ in $[10]$. Our result obtained extends Neubrander's one [7] on the special case of (SE) where $A(t)=A$ is independent of $t$ and $A$ is the infinitesimal generator of a $\left(C_{0}\right)$-semigroup on $X$ ( and so $D$ is dense in $X$ ).

In what follows, for a Banach space $X_{i}(i=1,2)$, the associated norm is denoted by $\|\cdot\|_{X_{i}}$ and $L\left(X_{1}, X_{2}\right)$ denotes the Banach space of all bounded linear operators from $X_{1}$ into $X_{2}$. The norm in $L\left(X_{1}, X_{2}\right)$ is denoted by $\|\cdot\|_{X_{1}, X_{2}}$.

Received by the editors January 17, 1995 and, in revised form, April 3, 1995.

1991 Mathematics Subject Classification. Primary 42D05, 34G10. 


\section{The Results on $\left(\mathrm{CP} ; u_{0}, f\right)$}

In this section we recall the fundamental results obtained by Tanaka [10] on the problem

$\left(\mathrm{CP} ; u_{0}, f\right)$

$$
\left\{\begin{array}{l}
u^{\prime}(t)=A(t) u(t)+f(t) \text { for } t \in[0, T] \\
u(0)=u_{0}
\end{array}\right.
$$

and introduce the notion of a $D$-integral solution of $\left(\mathrm{CP} ; u_{0}, f\right)$.

Here $D$ is another Banach space with norm $\|\cdot\|_{D}$ which is continuously embedded in $X$. Assume the following three conditions on a family $\{A(t): t \in[0, T]\}$ of closed linear operators in $X$.

(a1) $D(A(t))=D$ is independent of $t$, and there exists a $c_{0}>0$ such that

$$
c_{0}^{-1}\|y\|_{D} \leq\|y\|_{X}+\|A(t) y\|_{X} \leq c_{0}\|y\|_{D}
$$

for $y \in D$ and $t \in[0, T]$.

(a2) There are constants $M \geq 1$ and $\omega \in(-\infty, \infty)$ such that

$$
(\omega, \infty) \subset \rho(A(t)) \text { for } t \in[0, T]
$$

and

$$
\left\|\prod_{j=1}^{k}\left(\lambda I-A\left(t_{j}\right)\right)^{-1}\right\| \leq M(\lambda-\omega)^{-k} \text { for } \lambda>\omega
$$

and every finite sequence $\left\{t_{j}\right\}_{j=1}^{k}$ with $0 \leq t_{1} \leq t_{2} \leq \cdots \leq t_{k} \leq T$ and $k=1,2, \cdots$.

Then by $(2.1)$ and $(2.2)$ for $\lambda_{0}>\omega_{0}:=\max (0, \omega)$ there exists a $c>0$ such that

$$
\left\|\left(A(t)-\lambda_{0}\right)^{-1} x\right\|_{D} \leq c\|x\|_{X} \text { for } x \in X .
$$

(a3) For $y \in D, A(t) y$ is continuously differentiable in $X$.

First recall the results in $[10]$ on the problem $\left(\mathrm{CP} ; u_{0}, f\right)$.

By [10, Theorem 4.2] we obtain the following existence and uniqueness result of classical solutions of the problem $\left(\mathrm{CP} ; u_{0}, f\right)$.

Theorem 2.1. Suppose conditions (a1)-(a3). Let $f \in W^{1,1}([0, T]: X)$ and suppose that $u_{0} \in D$ satisfies the compatibility condition that $A(0) u_{0}+f(0) \in \bar{D}$. Then $\left(\mathrm{CP} ; u_{0}, f\right)$ has a unique classical solution $u \in C([0, T]: D) \cap C^{1}([0, T]: X)$ given by

$$
u(t)=\lim _{\lambda \rightarrow 0+}\left(U_{\lambda}(t, 0) u_{0}+\int_{0}^{[t / \lambda] \lambda} U_{\lambda}(t, s) f(s) d s\right)
$$

for $t \in[0, T]$, where $U_{\lambda}(t, s)=\prod_{i=[s / \lambda]+1}^{[t / \lambda]}(I-\lambda A(i \lambda))^{-1}$ for $0 \leq s \leq t \leq T$.

Moreover $u$ satisfies the integral equation

$$
\begin{aligned}
& \left(A(t)-\lambda_{0}\right) u(t)+f(t)=U(t, 0)\left(\left(A(0)-\lambda_{0}\right) u_{0}+f(0)\right) \\
& \quad+\lim _{\lambda \rightarrow 0+} \int_{0}^{[t / \lambda] \lambda} U_{\lambda}(t, s)\left(\dot{A}(s) u(s)+f^{\prime}(s)-\lambda_{0} f(s)\right) d s
\end{aligned}
$$

for $t \in[0, T]$, where $\lambda_{0}>\omega_{0}$ and $U(t, 0) z=\lim _{\lambda \rightarrow 0+} U_{\lambda}(t, 0) z$ for $z \in \bar{D}$ and $t \in[0, T]$ (see (4.2) and (4.5) in [10]). 
Remark 2.1. The combination of (2.4) and (2.5) gives

$$
\begin{aligned}
A(t) u(t)+f(t) & =U(t, 0)\left(A(0) u_{0}+f(0)\right) \\
& +\lim _{\lambda \rightarrow 0+} \int_{0}^{[t / \lambda] \lambda} U_{\lambda}(t, s)\left(\dot{A}(s) u(s)+f^{\prime}(s)\right) d s
\end{aligned}
$$

for $t \in[0, T]$.

One integrates $\left(\mathrm{CP} ; u_{0}, f\right)$ formally and then an integration by parts yields

$$
\begin{aligned}
u(t)-u_{0} & =\int_{0}^{t} A(s) u(s) d s+\int_{0}^{t} f(s) d s \\
& =A(t) \int_{0}^{t} u(s) d s-\int_{0}^{t} \dot{A}(s) \int_{0}^{s} u(r) d r d s+\int_{0}^{t} f(s) d s .
\end{aligned}
$$

This consideration leads us to the following definition.

Definition 2.1. Let $u_{0} \in X$ and $f \in L^{1}([0, T]: X)$. A function $u:[0, T] \rightarrow X$ is called a $D$-integral solution of $\left(\mathrm{CP} ; u_{0}, f\right)$ if $u \in C([0, T]: X), \int_{0}^{*} u(s) d s \in C([0, T]$ : $D)$ and

$$
u(t)-u_{0}=A(t) \int_{0}^{t} u(s) d s-\int_{0}^{t} \dot{A}(s) \int_{0}^{s} u(r) d r d s+\int_{0}^{t} f(s) d s .
$$

Remark 2.2. The notion of a $D$-integral solution of $\left(\mathrm{CP} ; u_{0}, f\right)$ coincides with that of an integral solution introduced by Da Prato and Sinestrari [1] in the case where $A(t)=A$ is independent of $t$.

Let $u_{0} \in \bar{D}$ and $f \in L^{1}([0, T]: X)$. Then it is shown in [10, Theorem 3.1] that the limit (2.4) exists uniformly in $t \in[0, T]$. Then we have

Theorem 2.2. Suppose conditions (a1)-(a3). Let $u_{0} \in \bar{D}$ and $f \in L^{1}([0, T]$ : $X)$. A function $u$ defined by $(2.4)$ is a unique $D$-integral solution of $\left(\mathrm{CP} ; u_{0}, f\right)$. Moreover we have the following estimates:

$$
\begin{aligned}
& \|u(t)\|_{X} \leq C_{1}\left(\left\|u_{0}\right\|_{X}+\int_{0}^{t}\|f(s)\|_{X} d s\right) ; \\
& \left\|\int_{0}^{t} u(s) d s\right\|_{D} \leq C_{1}\left(\left\|u_{0}\right\|_{X}+\int_{0}^{t}\|f(s)\|_{X} d s\right)
\end{aligned}
$$

for $t \in[0, T]$, where $C_{1}$ is a constant independent of $u_{0}$ and $f$.

Proof. Let $v_{0}(t)=0$ on $t \in[0, T]$. Since $u_{0} \in \bar{D}$, by Theorem 2.1 we can define $v_{n} \in C^{1}([0, T]: X) \cap C([0, T]: D)$ inductively by the unique classical solution of the problem

$$
\left\{\begin{array}{l}
v_{n}^{\prime}(t)=A(t) v_{n}(t)+u_{0}-\int_{0}^{t} \dot{A}(s) v_{n-1}(s) d s+\int_{0}^{t} f(s) d s \text { for } t \in[0, T] \\
v_{n}(0)=0
\end{array}\right.
$$

for $n \geq 1$. 
Then from (2.5) it follows that

$$
\begin{aligned}
&\left(A(t)-\lambda_{0}\right) v_{n}(t)+u_{0}-\int_{0}^{t} \dot{A}(s) v_{n-1}(s) d s+\int_{0}^{t} f(s) d s \\
&=U(t, 0) u_{0}+\lim _{\lambda \rightarrow 0+} \int_{0}^{[t / \lambda] \lambda} U_{\lambda}(t, s)\left[\dot{A}(s) v_{n}(s)-\dot{A}(s) v_{n-1}(s)+f(s)\right. \\
&\left.\quad-\lambda_{0}\left(u_{0}-\int_{0}^{s} \dot{A}(r) v_{n-1}(r) d r+\int_{0}^{s} f(r) d r\right)\right] d s
\end{aligned}
$$

for $t \in[0, T]$. This together with (2.3) shows that there exists a constant $K>0$ such that

$$
\left\|v_{n+1}(t)-v_{n}(t)\right\|_{D} \leq K \int_{0}^{t}\left(\left\|v_{n+1}(s)-v_{n}(s)\right\|_{D}+\left\|v_{n}(s)-v_{n-1}(s)\right\|_{D}\right) d s,
$$

which implies by Gronwall's inequality that

$$
\left\|v_{n+1}(t)-v_{n}(t)\right\|_{D} \leq K \int_{0}^{t} e^{K(t-s)}\left\|v_{n}(s)-v_{n-1}(s)\right\|_{D} d s
$$

for $t \in[0, T]$ and $n \geq 1$. By standard arguments we see that there exists an element $v \in C([0, T]: D)$ such that $\sup \left\{\left\|v_{n}(t)-v(t)\right\|_{D}: t \in[0, T]\right\}$ converges to zero as $n \rightarrow \infty$. Thus $v_{n}^{\prime}(t)\left(=A(t) v_{n}(t)+u_{0}-\int_{0}^{t} \dot{A}(s) v_{n-1}(s) d s+\int_{0}^{t} f(s) d s\right)$ converges to $A(t) v(t)+u_{0}-\int_{0}^{t} \dot{A}(s) v(s) d s+\int_{0}^{t} f(s) d s$ uniformly in $t \in[0, T]$ as $n \rightarrow \infty$ and hence $v^{\prime}$ is a $D$-integral solution of $\left(\mathrm{CP} ; u_{0}, f\right)$.

Let $w$ be a $D$-integral solution of $\left(\mathrm{CP} ; u_{0}, f\right)$ and let $w_{1}(t)=\int_{0}^{t} w(s) d s$. Then since $w_{1}^{\prime}(t)=A(t) w_{1}(t)+u_{0}-\int_{0}^{t} \dot{A}(s) w_{1}(s) d s+\int_{0}^{t} f(s) d s$ and $w_{1}(0)=0$, by $(2.6)$ we have

$$
\begin{aligned}
w(t) & =w_{1}^{\prime}(t) \\
& =U(t, 0) u_{0}+\lim _{\lambda \rightarrow 0+} \int_{0}^{[t / \lambda] \lambda} U_{\lambda}(t, s)\left\{\dot{A}(s) w_{1}(s)+\left(-\dot{A}(s) w_{1}(s)+f(s)\right)\right\} d s
\end{aligned}
$$

which is equal to a function $u$ defined by (2.4). This proves the uniqueness of $D$-integral solutions of $\left(\mathrm{CP} ; u_{0}, f\right)$. This together with $(2.4)$ shows $(2.8)$.

Finally we shall show (2.9). By (2.1), (2.7) and (2.8) we get

$$
\begin{aligned}
c_{0}^{-1} \| & \int_{0}^{t} u(s) d s\left\|_{D} \leq\right\| \int_{0}^{t} u(s) d s\left\|_{X}+\right\| A(t) \int_{0}^{t} u(s) d s \|_{X} \\
& \leq \int_{0}^{t}\|u(s)\|_{X} d s+\left\|u(t)-u_{0}+\int_{0}^{t} \dot{A}(s) \int_{0}^{s} u(r) d r d s-\int_{0}^{t} f(s) d s\right\|_{X} \\
& \leq C_{2}\left(\left\|u_{0}\right\|_{X}+\int_{0}^{t}\|f(s)\|_{X} d s\right)+\int_{0}^{t}\|\dot{A}(s)\|_{D, X}\left\|\int_{0}^{s} u(r) d r\right\|_{D} d s
\end{aligned}
$$

for $t \in[0, T]$, where $C_{2}$ is a constant independent of $u_{0}$ and $f$. Thus Gronwall's inequality gives the desired estimate (2.9). 


\section{Complete SECOND ORDER LINEAR DIFFERENTIAL EQUATIONS}

Now we turn to the complete second order linear differential equations

$$
\left\{\begin{array}{l}
u^{\prime \prime}(t)=A(t) u^{\prime}(t)+B(t) u(t)+f(t) \text { for } t \in[0, T] \\
u(0)=x \text { and } u^{\prime}(0)=y .
\end{array}\right.
$$

A family $\{A(t): t \in[0, T]\}$ of closed linear operators in $X$ is assumed to satisfy conditions (a1) - (a3) in Section 2. We assume the next two conditions on a family $\{B(t): t \in[0, T]\}$ of linear operators in $X:$

(b1) For $t \in[0, T], D \subset D(B(t))$ and $B(t) \in L(D, X)$.

(b2) For $y \in \cap_{0 \leq t \leq T} D(B(t)), B(t) y$ is continuously differentiable in $X$.

It should be noted that we do not assume the closedness of the operator $B(t)$ in $X$.

Definition 3.1. A function $u:[0, T] \rightarrow X$ is called a D-classical solution of (SE) if $u \in C^{2}([0, T]: X), u(t) \in D(B(t)), u^{\prime}(t) \in D$, the functions $B(t) u(t), A(t) u^{\prime}(t)$ are continuous in $t$ and $u$ satisfies (SE).

Let $x \in \cap_{0 \leq t \leq T} D(B(t))$. Consider the following first order differential equation:

$$
\left\{\begin{array}{l}
u^{\prime}(t)=A(t) u(t)+B(t) x+B(t) \int_{0}^{t} u(s) d s+f(t) \text { for } t \in[0, T] \\
u(0)=y
\end{array}\right.
$$

We say that $u$ is a $D$-classical solution of $(\mathrm{FE})$ if $u \in C^{1}([0, T]: X) \cap C([0, T]: D)$ and $u$ satisfies (FE).

Remark 3.1. If $u \in C([0, T]: D)$, then $\int_{0}^{\cdot} u(s) d s \in C([0, T]: D)$ by (a1) and the closedness of $A(0)$.

By assumptions (b1) and (b2) we obtain the following proposition immediately.

Proposition 3.1. Suppose conditions (a1)-(a3) and (b1)-(b2) are fulfilled. A function $u:[0, T] \rightarrow X$ is a D-classical solution of $(\mathrm{FE})$ if and only if $v(\cdot)$ defined by $v(t)=x+\int_{0}^{t} u(s) d s$ is a D-classical solution of $(\mathrm{SE})$.

Now we are in a position to state our main result in this paper.

Theorem 3.2. Suppose conditions (a1)-(a3) and (b1)-(b2) are fulfilled. Let $f \in$ $W^{1,1}([0, T]: X), x \in \cap_{0 \leq t \leq T} D(B(t))$ and $y \in D$. If the compatibility condition that $B(0) x+A(0) y+f(0) \in \bar{D}$ is satisfied, then (SE) has a unique D-classical solution $v$ which satisfies

$$
\|v(t)\|_{X} \leq C\left(\|x\|_{X}+\|y\|_{X}+\int_{0}^{t}\|B(s) x+f(s)\|_{X} d s\right)
$$

for $t \in[0, T]$, where $C$ is a constant independent of $x, y$ and $f$.

Proof. By Proposition 3.1, it is sufficient to prove the existence and uniqueness of $D$-classical solutions of $(\mathrm{FE})$.

Let $u_{0}(t)=y$ for $t \in[0, T]$. By Theorem 2.1 we can define

$$
u_{n} \in C^{1}([0, T]: X) \cap C([0, T]: D)
$$


inductively by the unique classical solution of the problem

$$
\left\{\begin{array}{l}
u_{n}^{\prime}(t)=A(t) u_{n}(t)+B(t) x+B(t) \int_{0}^{t} u_{n-1}(s) d s+f(t) \text { for } t \in[0, T] \\
u_{n}(0)=y
\end{array}\right.
$$

for $n \geq 1$.

Then by the same arguments used in proving Theorem 2.2 we see that $u_{n}$ converges to an element $u$ in $C([0, T]: D)$ as $n \rightarrow \infty$ in the topology of $C([0, T]: D)$ and that $u$ is a $D$-classical solution of (FE).

To prove the uniqueness of $D$-classical solutions of $(\mathrm{FE})$, let $u_{i}(i=1,2)$ be $D$ classical solutions of $(\mathrm{FE})$ and let $w=u_{1}-u_{2}$. Then we have $w^{\prime}(t)=A(t) w(t)+$ $B(t) \int_{0}^{t} w(s) d s$ and $w(0)=0$, and so by $(2.5)$

$$
\begin{aligned}
\left(A(t)-\lambda_{0}\right) w(t)+B(t) \int_{0}^{t} w(s) d s & \\
=\lim _{\lambda \rightarrow 0+} \int_{0}^{[t / \lambda] \lambda} U_{\lambda}(t, s) & {[\dot{A}(s) w(s)} \\
& \left.\quad+B(s) w(s)+\dot{B}(s) \int_{0}^{s} w(r) d r-\lambda_{0} B(s) \int_{0}^{s} w(r) d r\right] d s
\end{aligned}
$$

for $t \in[0, T]$. The estimation of this equality gives

$$
\|w(t)\|_{D} \leq K^{\prime} \int_{0}^{t}\|w(s)\|_{D} d s
$$

for some constant $K^{\prime}>0$. By Gronwall's inequality we have $w=0$.

Finally we shall show the estimate (3.1).

Since $u$ is a $D$-integral solution of $\left(\mathrm{CP} ; y, B(\cdot) x+B(\cdot) \int_{0}^{\cdot} u(s) d s+f(\cdot)\right)$, the estimate (2.9) gives

$$
\begin{aligned}
\left\|\int_{0}^{t} u(s) d s\right\|_{D} \leq & C_{1}\left(\|y\|_{X}+\int_{0}^{t}\left\|B(s) x+B(s) \int_{0}^{s} u(r) d r+f(s)\right\|_{X} d s\right) \\
\leq & C_{1}\left(\|y\|_{X}+\int_{0}^{t}\|B(s) x+f(s)\|_{X} d s\right) \\
& +C_{1} \int_{0}^{t}\|B(s)\|_{D, X}\left\|\int_{0}^{s} u(r) d r\right\|_{D} d s,
\end{aligned}
$$

which implies by Gronwall's inequality that

$$
\left\|\int_{0}^{t} u(s) d s\right\|_{D} \leq C_{3}\left(\|y\|_{X}+\int_{0}^{t}\|B(s) x+f(s)\|_{X} d s\right)
$$

for some constant $C_{3}>0$. Combining this with (2.8) we obtain

$$
\begin{aligned}
\|u(t)\|_{X} & \leq C_{1}\left(\|y\|_{X}+\int_{0}^{t}\left\|B(s) x+B(s) \int_{0}^{s} u(r) d r+f(s)\right\|_{X} d s\right) \\
& \leq C_{4}\left(\|y\|_{X}+\int_{0}^{t}\|B(s) x+f(s)\|_{X} d s\right)
\end{aligned}
$$

for some constant $C_{4}>0$. Therefore Proposition 3.1 implies the desired estimate (3.1). 
The rest of this section is devoted to an application of Theorem 3.2 to the following hyperbolic partial differential equation :

$$
\text { (P) }\left\{\begin{array}{r}
u_{t t}+\alpha(t, x) u_{t x}+\beta(t, x) u_{t}=\gamma(t, x) u_{x}+\delta(t, x) u+f(t, x), \\
\quad(t, x) \in[0, T] \times[0,1], \\
u(t, 0)=u(t, 1), t \in[0, T], u(0, x)=u_{0}(x), u_{t}(0, x)=u_{1}(x), x \in[0,1] .
\end{array}\right.
$$

We denote by $X$ the Banach space $C[0,1]$ with norm $\|u\|_{\infty}=\sup \{|u(x)|$ : $x \in[0,1]\}$ and by $D$ the Banach space $\left\{u \in C^{1}[0,1]: u(0)=u(1)\right\}$ with norm $\|u\|_{\infty}+\left\|u^{\prime}\right\|_{\infty}$. Define three families $\{A(t): t \in[0, T]\},\{B(t): t \in[0, T]\}$ and $\{C(t): t \in[0, T]\}$ of linear operators in $X$ by

$$
\begin{aligned}
& \left\{\begin{array}{l}
D(A(t))=D \\
(A(t) u)(x)=-\alpha(t, x) u^{\prime}(x) \text { for } u \in D,
\end{array}\right. \\
& \left\{\begin{array}{l}
D(B(t))=C^{1}[0,1] \\
(B(t) u)(x)=\gamma(t, x) u^{\prime}(x)+\delta(t, x) u(x) \text { for } u \in C^{1}[0,1], \text { and }
\end{array}\right. \\
& \left\{\begin{array}{l}
D(C(t))=X \\
(C(t) u)(x)=-\beta(t, x) u(x) \text { for } u \in X,
\end{array}\right.
\end{aligned}
$$

respectively.

If $\alpha$ is a positive function of class $C^{1}$, then the family $\{A(t): t \in[0, T]\}$ of closed linear operators satisfies three conditions (a1) - (a3) (see [2, Theorem 6.1]). Also, it is well-known that the stability condition is preserved under the perturbation of a uniformly bounded family of bounded linear operators on $X$ (see [4] or [9, Theorem 5.2.3]). Therefore, if $\beta$ is of class $C^{1}$, the family $\{A(t)+C(t): t \in[0, T]\}$ of closed linear operators in $X$ satisfies conditions (a1) - (a3) with $A(t)$ replaced by $A(t)+C(t)$. It is easy to see that conditions (b1) - (b2) are satisfied if $\gamma$ and $\delta$ are of class $C^{1}$. Since $\bar{D}=\{u \in C[0,1]: u(0)=u(1)\}$, Theorem 3.2 asserts that if $f \in W^{1,1}([0, T]: C[0,1]), u_{0}, u_{1} \in C^{1}[0,1], u_{1}(0)=u_{1}(1)$ and the compatibility condition that $-\alpha(0,0) u_{1}^{\prime}(0)-\beta(0,0) u_{1}(0)+\gamma(0,0) u_{0}^{\prime}(0)+\delta(0,0) u_{0}(0)+f(0,0)=$ $-\alpha(0,1) u_{1}^{\prime}(1)-\beta(0,1) u_{1}(1)+\gamma(0,1) u_{0}^{\prime}(1)+\delta(0,1) u_{0}(1)+f(0,1)$ is satisfied, then the problem $(\mathrm{P})$ has a unique solution $u \in C^{2}([0, T]: C[0,1]) \cap C\left([0, T]: C^{1}[0,1]\right)$ which satisfies the estimate :

$$
\begin{aligned}
\sup _{x \in[0,1]}|u(t, x)| \leq & C\left(\sup _{x \in[0,1]}\left|u_{0}(x)\right|+\sup _{x \in[0,1]}\left|u_{1}(x)\right|\right. \\
& \left.+\int_{0}^{t} \sup _{x \in[0,1]}\left|\gamma(s, x) u_{0}^{\prime}(x)+\delta(s, x) u_{0}(x)+f(s, x)\right| d s\right)
\end{aligned}
$$

for $t \in[0, T]$, where $C$ is a constant independent of $u_{0}, u_{1}$ and $f$.

\section{REFERENCES}

[1] G. Da Prato and E. Sinestrari, Differential operators with non dense domain, Ann. Scuola Norm. Sup. Pisa XIV (1987), 285-344. MR 89f:47062

[2] G. Da Prato and E. Sinestrari, Non autonomous evolution operators of hyperbolic type, Semigroup Forum 45 (1992), 302-321. MR 93h:34101

[3] R. Grimmer and J.H. Liu, Integrated semigroups and integrodifferential equations, Semigroup Forum 48 (1994), 79-95. MR 95f:47066 
[4] T. Kato, Linear evolution equations of "hyperbolic" type, J. Fac. Sci. Univ. Tokyo, Sec. I 17 (1970), 241-258. MR 43:5347

[5] S.G. Krein, "Linear Differential Equations in Banach Spaces," Translations of Math. Monographs, Vol. 29, Amer. Math. Soc., Providence, R I. 1971. MR 49:2548

[6] R. Nagel and E. Sinestrari, Inhomogeneous Volterra integrodifferential equations for HilleYosida operators. In: K.D. Bierstedt, A. Pietsch, W.M. Ruess, D. Vogt (eds.) : Functional Analysis, Proc. Essen Conference. Lect. Notes Pure Appl. Math. 150, Marcel Dekker (1994), 51-70. MR 94i:34121

[7] F. Neubrander, Well-posedness of higher order abstract Cauchy problems, Trans. Amer. Math. Soc. 295 (1986), 257-290. MR 88a:34087

[8] F. Neubrander, Integrated semigroups and their application to complete second order Cauchy problems, Semigroup Forum 38 (1989), 233-251. MR 89m:47038

[9] A. Pazy, "Semigroups of Linear Operators and Applications to Partial Differential Equations,"Springer-Verlag, New York, 1983. MR 85g:47061

[10] N. Tanaka, Semilinear equations in the "hyperbolic" case, Nonlinear Analysis 24 (1995), $773-788$

School of Education, Department of Mathematics, Waseda University, 1-6-1 NishiWASEDA, ShinJuku-Ku, TOKYo 169-50, JaPan

Current address: Ibaraki University, Faculty of Engineering, 12-1 Nakanarusawa 4 chome, Hitachi, Ibaraki, 316 Japan

E-mail address: oka@base.ibaraki.ac.jp 